\title{
Anomalous Absorption in Arrays of Metallic Nanoparticles: A Powerful Tool for Quantum Dot Optoelectronics
}

\author{
Augustin Caillas ${ }^{1}$, Stéphan Suffit ${ }^{1}$, Pascal Filloux $^{1}$, Emmanuel Lhuillier $^{2}$, Aloyse Degiron ${ }^{1, *}$ \\ ${ }^{1}$ Université de Paris, CNRS, Laboratoire Matériaux et Phénomènes Quantiques, F-75013 Paris, France \\ ${ }^{2}$ Sorbonne Université, CNRS, Institut des NanoSciences de Paris, INSP, F-75005 Paris, France \\ *aloyse.degiron@u-paris.fr
}

\section{Supporting Information}

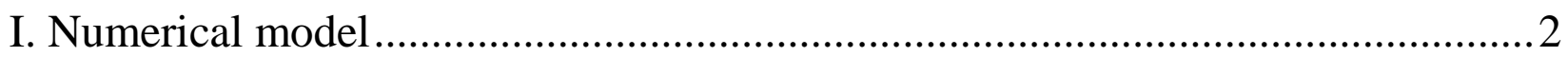

II. Numerical simulations with a much smaller filling factor ..................................

III. Analysis of the fields and associated currents when EIA occurs....................... 4

IV. How to design the metasurfaces to operate at other wavelengths .....................6

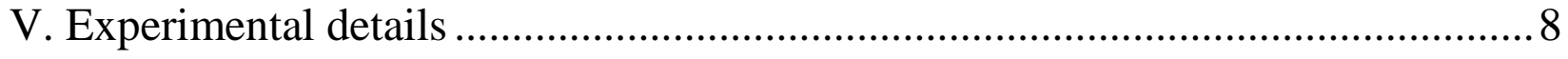

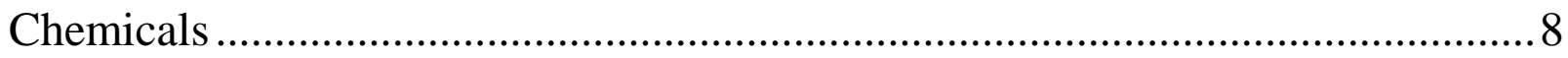

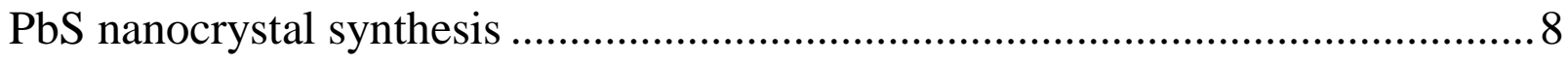

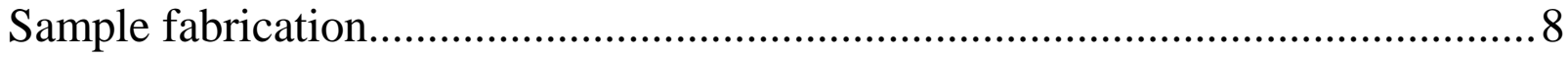

Photoluminescence experiments ................................................................ 10

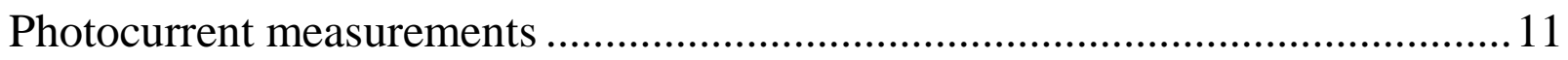

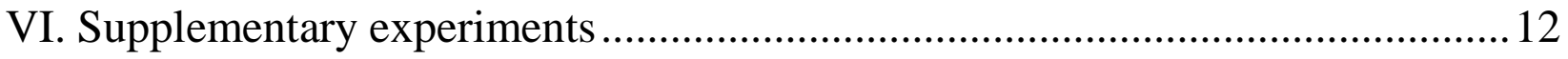

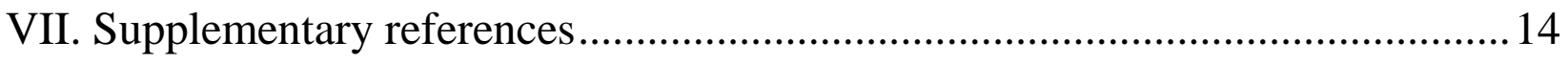




\section{Numerical model}

The structures are simulated using the RF module of Comsol Multiphysics, a finite element solver. The model studied in Figure 1 consists of a single unit cell flanked with periodic conditions. The bottom half of the unit cell has a height of $3 \mu \mathrm{m}$ and is filled with glass with a refractive index $n_{\mathrm{g}}=$ 1.45. This index corresponds to the experimental value of our Borofloat glass substrate from Plan Optik, measured by fitting the diffraction orders observed in some of our PL maps with the light lines $\omega= \pm \mathrm{k}_{\mathrm{x}} \mathrm{c} / \mathrm{n}_{\mathrm{g}} \mp 2 \pi \mathrm{c} /\left(\mathrm{n}_{\mathrm{g}} \mathrm{P}\right)$. The upper half is also $3-\mu \mathrm{m}$ high and is filled with air $(\mathrm{n}=1)$. At the interface between these two media, the Au antennas are modelled with a permittivity taken from the experimental literature. ${ }^{1}$ Electromagnetic waves enter and exit the model through ports applied on the top and bottom of the computational domain. The number of ports on each side is chosen so as to take all diffraction orders into account. The port associated with the zeroth diffraction order on the air side is used to launch a TE- or TM-polarized wave at a specified incidence angle.

The simulations presented in Figure 2a utilize a slightly modified version of the model to account for the actual dimensions of the Au squares (415 nm rather than $400 \mathrm{~nm}$, see Figure S7) and for the presence of the $\mathrm{PbS}$ nanocrystals (NCs) covering the sample. By systematic comparisons between experiments and simulations, we have noticed that the influence of the PbS NCs on the optical properties of the arrays can be modeled in a satisfying manner by burying the square nanoparticles $10 \mathrm{~nm}$ below the surface of the glass substrate (the refractive index is still $\mathrm{n}_{\mathrm{g}}=1.45$ without imaginary part). We have verified that this approximation does not only work for the specific

structure studied in the article, but also for other metasurfaces operating at other wavelengths, as can be appreciated for example on Figure S12.

\begin{tabular}{|c|l|}
\hline Air & $\begin{array}{l}\text { Figure S1. Schematic } \\
\text { cross-section of the } \\
\text { model used to simulate a } \\
\text { metasurface unit cell } \\
\text { class }\end{array}$ \\
covered with a thin layer \\
of PbS NCs.
\end{tabular}




\section{Numerical simulations with a much smaller filling factor}

In these simulations, the period $\mathrm{P}=600 \mathrm{~nm}$ is the same as in the main text but the lateral size of the Au particles has been reduced to $200 \mathrm{~nm} \times 200 \mathrm{~nm}$ (the Au thickness has been kept constant at $25 \mathrm{~nm}$ ). All other conditions are the same as in Figure 2. Contrarily to the EIA case studied in the main text, there is no dip in the reflectance peak for the TE polarization. Moreover, the absorption maxima are essentially similar to those seen on the reflectance map, which is the standard and expected behavior of a nanoparticle array with optical properties dominated by plasmonic resonances localized on each Au inclusion. Note also that these plasmonic resonances are shifted to higher frequencies due to the smaller size of the Au nanoparticles and that they are strongly modulated by the diffraction orders that arise above the light lines.
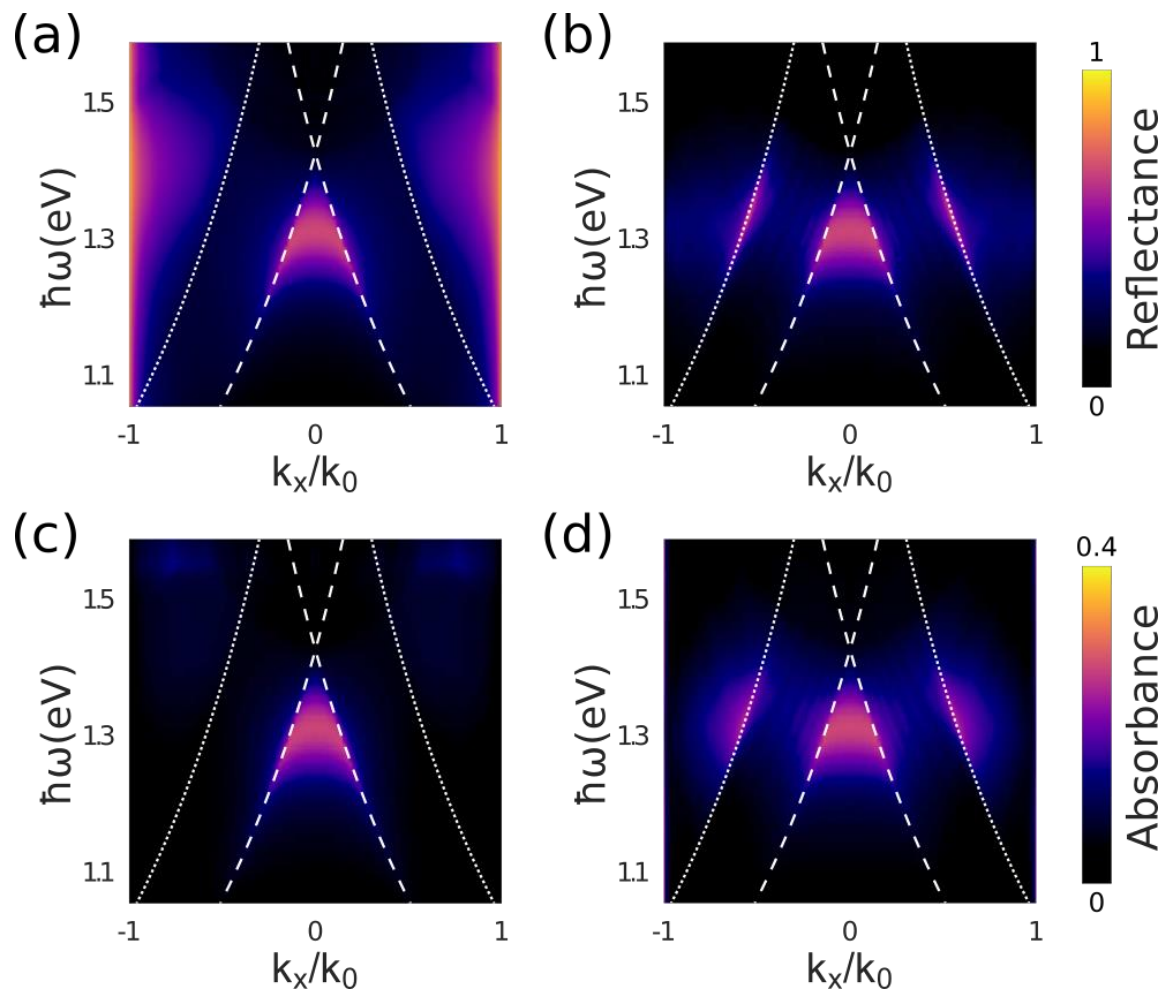

Figure S2. Simulated optical properties of the array when the size of the square nanoparticles is reduced to $200 \mathrm{~nm} \times 200 \mathrm{~nm} \times 25 \mathrm{~nm}$. (a)-(b) Simulated reflectance dispersion relation for the TE and TM polarizations. (c)-(d) Simulated absorbance for the TE and TM polarizations. The dashed and dotted white lines are the light lines in the glass substrate and air, respectively. 


\section{Analysis of the fields and associated currents when EIA occurs}
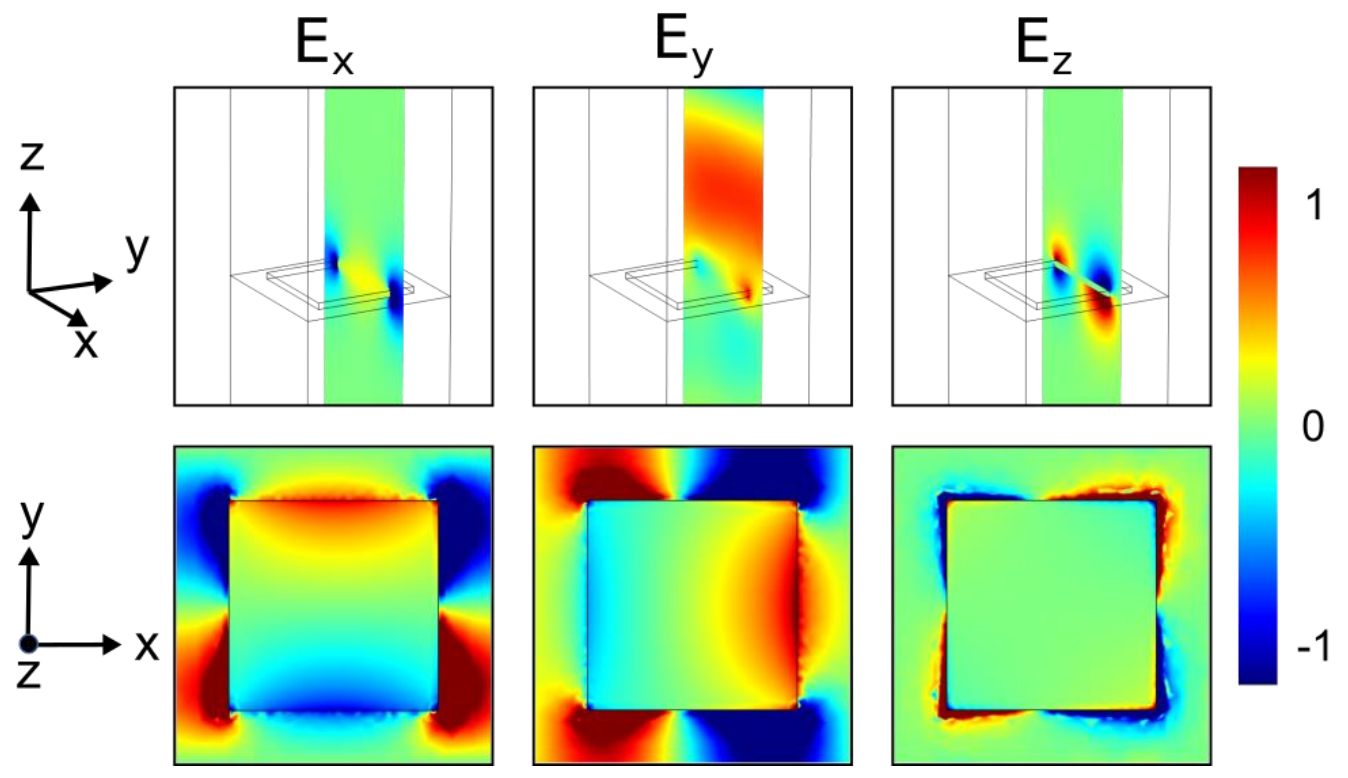

Figure S3. Plots of the $E_{x}, E_{y}$ and $E_{z}$ electric field components in the $x-z$ plane (top row) and $x-y$ plane (bottom row) at the wavelength of the EIA resonance $(\lambda=1280 \mathrm{~nm})$. The colors have been saturated to improve the visibility of the field symmetries. The structure shown here is the same as in Figure 2.

The spatial variations of the three components of the instantaneous electric field at the EIA wavelength are plotted in Figure S3 in two orthogonal planes. The positive (resp. negative) values mean that these $x, y$ or $z$ components are oriented along the positive (resp. negative) $x$-, $y$ - or $z$ axes. From these maps, it is possible to deduce a snapshot of the charge distribution, based on the consideration that the electric field lines, which are tangential to the electric field vector at all points, always originate from positive charges and terminate at negative charges: 


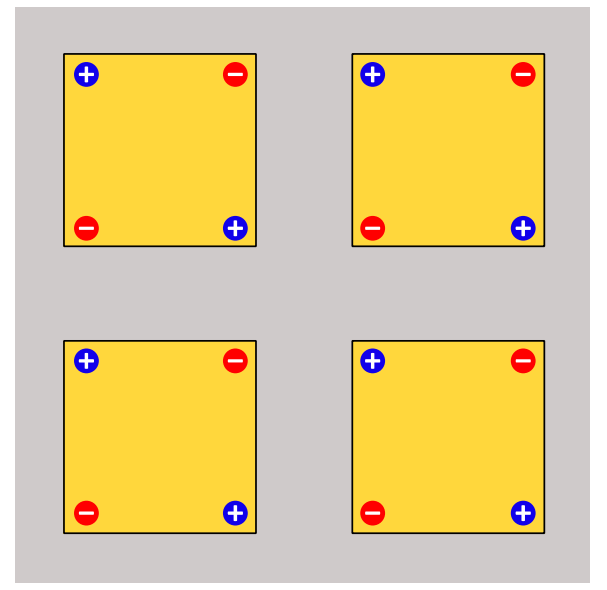

Figure S4. Schematic of the charges in the $x-y$ plane.

The associated current distribution are the loops sketched in the main text on Figure 1b. Note that these current loops are formed by real currents flowing on the edges of the Au patches and displacement currents $\vec{\imath}_{\text {Disp }} \propto \partial \vec{D} / \partial t$ within and between the Au patches, as is well documented in the literature for related geometries such as pairs of metallic rods and fishnet metamaterials. ${ }^{2,3}$

To gain quantitative insight, we plot the actual current density lines predicted by the numerical model on Figure S5. Notice that current loops are only observed when the EIA peak occurs (case $\mathrm{B}$ in Figure S5). These loops are resonant because they disappear outside the EIA peak (cases A and C). Each loop has a mirror symmetry with respect to the horizontal $x$-axis. The loops do not possess a mirror symmetry in the other direction because the plane-of-incidence for oblique illumination is the $x-z$ plane (see notations on Figure 1 in the main text). 

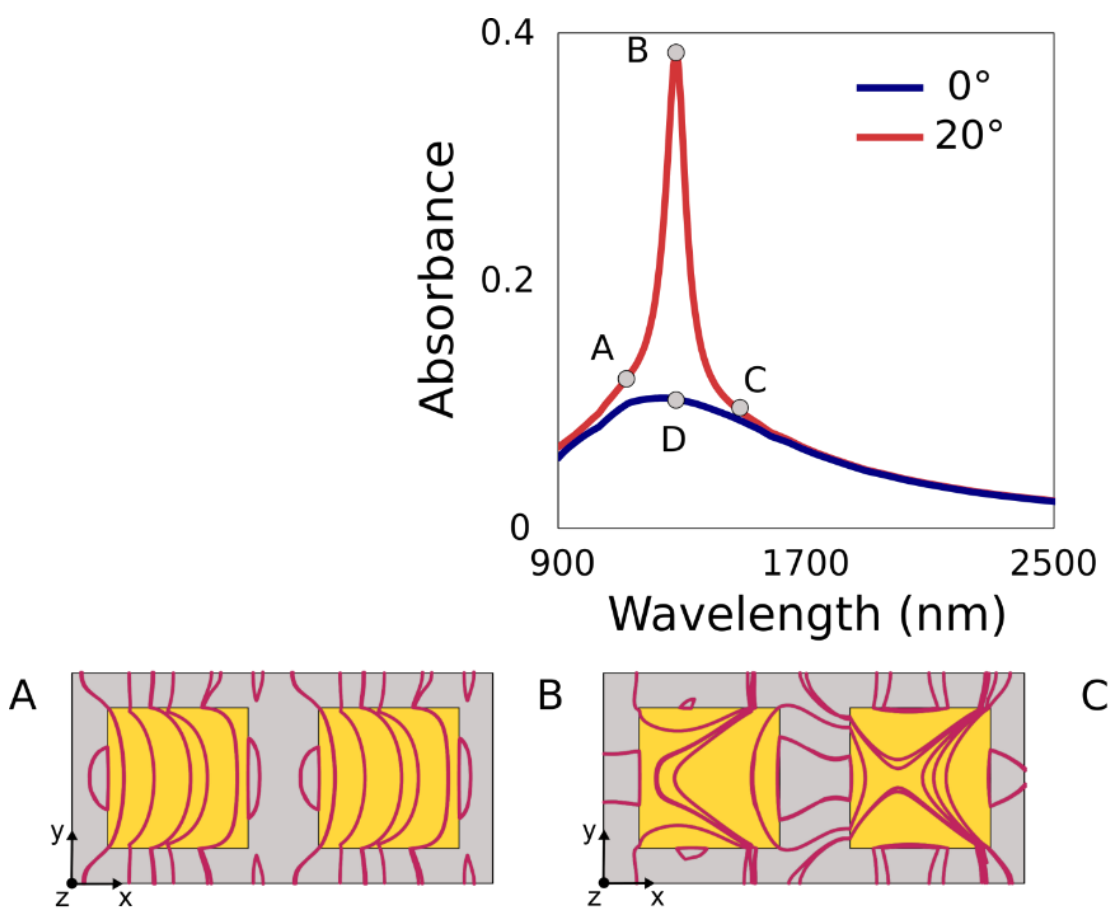

B

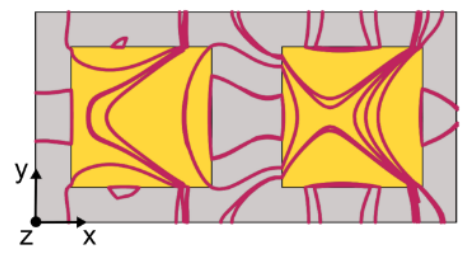

C

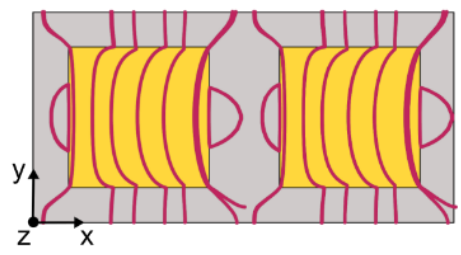

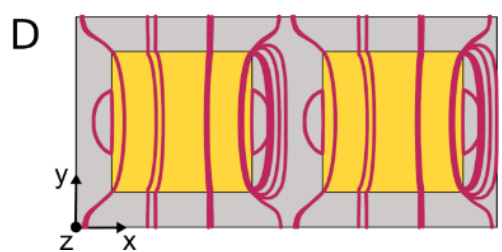

Figure S5. Current density lines computed for different wavelengths (A: $1120 \mathrm{~nm}, \mathrm{~B}$ and D: $1280 \mathrm{~nm}, \mathrm{C}$ : $1490 \mathrm{~nm}$ ) and different incidence angles (A, B \& C: illumination at $20^{\circ}$ with TE-polarized plane waves, D: illumination at normal incidence). The structure is the array of Au patches examined in Figure 2 in the main text. The absorbance spectra of Figure $2 \mathrm{~b}$ are reproduced here with gray marks identifying the four cases A, $\mathrm{B}, \mathrm{C}$ and $\mathrm{D}$.

\section{How to design the metasurfaces to operate at other wavelengths}

To produce a Fano response analogous to EIA, the plasmonic and LC resonances must overlap. The main geometrical parameter affecting the spectral position of the plasmonic resonance is the side of the Au patches because these plasmons are standing waves across the patches. However, this geometrical parameter also affects the size of the current loops between adjacent Au patches and thus the inductance of the LC resonances. In contrast, the period $\mathrm{P}$ of the system has a relatively 
small influence on the position of the plasmon resonances (their spectral width, however, is very sensitive to this parameter ${ }^{4}$ ) but it has a stronger impact on the capacitance of the LC resonance. In the case of an ideal lossless and perfectly conducting LC resonator, its resonance wavelength is proportional to $\sqrt{L C}$. This result, however, cannot be directly applied to our system due to the strong retardation effects occurring in metals at optical frequencies as well as the complex inhomogeneous environment in which the LC resonances develop. We have heuristically found that an efficient way to shift the EIA peak to smaller (resp. larger) wavelengths is to reduce (resp. increase) the side of the Au patches and the value of the period simultaneously, but not necessarily with a strict scaling factor, as illustrated on Figure S6.

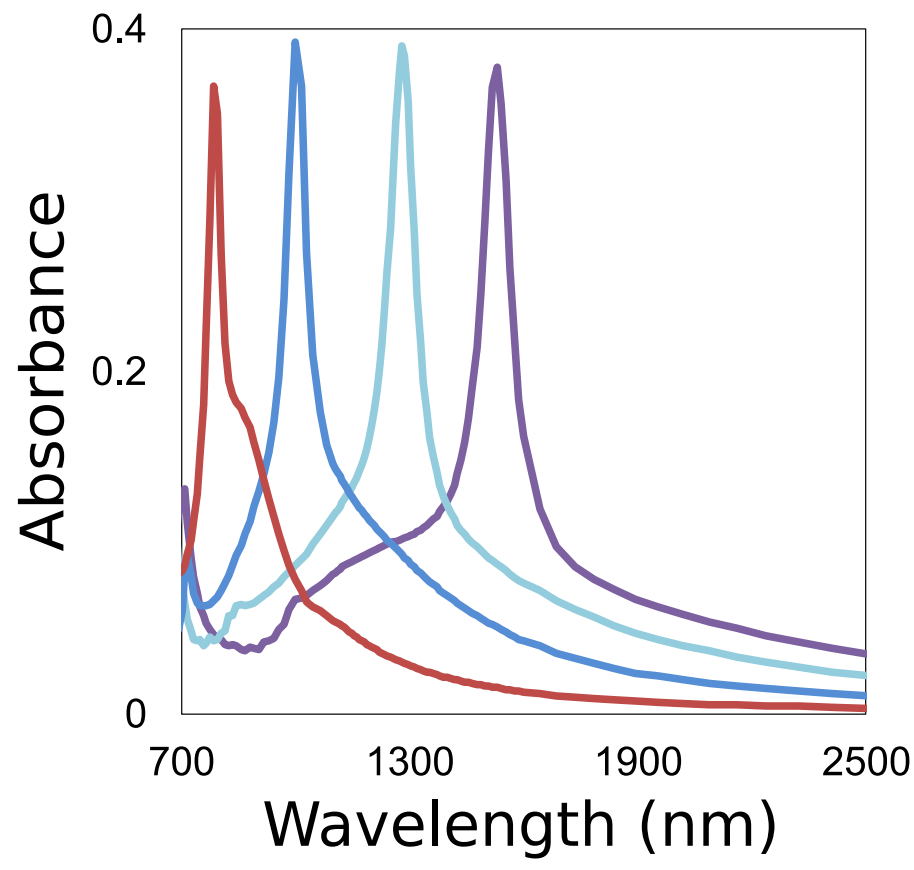

Figure S6. Numerical simulations of four different arrays of Au patches with different sides $\mathrm{S}$ and periods P. Red curve: $P=400 \mathrm{~nm}$ and $\mathrm{S}=200 \mathrm{~nm}$. Blue curve: $\mathrm{P}=500 \mathrm{~nm}$ and $\mathrm{S}=300 \mathrm{~nm}$. Light blue curve: $\mathrm{P}=$ $600 \mathrm{~nm}$ and $\mathrm{S}=400 \mathrm{~nm}$. Violet curve: $\mathrm{P}=700 \mathrm{~nm}$ and $\mathrm{S}=500 \mathrm{~nm}$. The metal thickness is $25 \mathrm{~nm}$ in all cases. All the curves have been computed for the TE polarization and an oblique illumination at $20^{\circ}$. 


\section{Experimental details}

\section{Chemicals}

Oleylamine (OLA, Acros, 80-90\%), \%), lead chloride $\left(\mathrm{PbCl}_{2}\right.$, Afla Aesar, $\left.99 \%\right)$, sulfur powder (S, Afla Aesar, 99.5\%), oleic acid (OA, Afla Aesar, 90\%), trioctylphosphine (TOP, Afla Aesar, 90\%), n-hexane (VWR), Ethanol (VWR, >99.9\%), Toluene (Carlo Erba, >99.8\%).

\section{PbS nanocrystal synthesis}

In a three-neck flask, $300 \mathrm{mg}$ of $\mathrm{PbCl}_{2}$, together with $100 \mu \mathrm{L}$ of TOP and $7.5 \mathrm{~mL}$ of OLA are degassed, first at room temperature and then at $110^{\circ} \mathrm{C}$ for $30 \mathrm{~min}$. Meanwhile, $30 \mathrm{mg}$ of S powder is mixed with $7.5 \mathrm{~mL}$ of OLA until full dissolution and an orange clear solution is obtained. Then under nitrogen at $80^{\circ} \mathrm{C}$, this solution of $\mathrm{S}$ is quickly added to the flask. After 2 minutes, the reaction is quickly quenched by addition of $1 \mathrm{~mL}$ of $\mathrm{OA}$ and $9 \mathrm{~mL}$ of hexane. The nanocrystals are precipitated with ethanol and redispersed in $5 \mathrm{~mL}$ of toluene. This washing step is repeated one more time and the pellet is this time dispersed in $10 \mathrm{~mL}$ of toluene with a drop of OA. The solution is then centrifugated as is, to remove the unstable phase. The supernatant is precipitated with methanol and redispersed in toluene. Finally, the $\mathrm{PbS} \mathrm{NC}$ solution in toluene is filtered through a $0.2 \mu \mathrm{m}$ PTFE filter. The obtained solution is used for further characterization and device fabrication.

\section{Sample fabrication}

The array of $\mathrm{Au}$ square nanoparticles studied in Figure 3a-3e is defined by electron-beam lithography on a borosilicate glass substrate from Plan Optik coated by a bilayer of CSAR 62 positive resist and Electra 92 conductive coating from Allresist. The conductive coating is applied to avoid a charge accumulation during the writing procedure, the latter being performed with a PIONEER Two Raith system. After deposition of $2 \mathrm{~nm}$ of Ti and $25 \mathrm{~nm}$ of Au in a Plassys MEB 
$550 \mathrm{~S}$ electron beam evaporator, followed by an overnight lift-off in butanone, the resulting $\mathrm{Au}$ nanoparticle array occupies an area of $200 \times 200 \mu \mathrm{m}^{2}$. The scanning electron micrograph (SEM) of Figure S7 reveals that the actual size of the square nanoparticles is $415 \mathrm{~nm} \times 415 \mathrm{~nm}$ instead of the nominal value of $400 \mathrm{~nm} \times 400 \mathrm{~nm}$. The measured period is $600 \mathrm{~nm}$, as expected.

The fabrication of the photoconductive detector studied in Figure $3 \mathrm{f}$ starts with a first level of optical lithography (using an MJB4 mask aligner and the AZ 5214E photoresist), metal deposition ( $2 \mathrm{~nm}$ of $\mathrm{Ti}$ and $70 \mathrm{~nm}$ of $\mathrm{Au}$ ) and lift-off to define the interdigitated electrodes. The spacing between two consecutive digits is $10 \mu \mathrm{m}$. These $10 \mu \mathrm{m}$ wide channels are then filled with arrays of Au nanoparticles following the fabrication steps described above in this section. The total area of the photoconductive device is $870 \times 1400 \mu \mathrm{m}^{2}$.

After defining the periodic arrays with and without interdigitated electrodes, the sample is introduced in a $\mathrm{N}_{2}$-filled glovebox. A $15-20 \mathrm{~nm}$ thick layer of NCs is spun onto the sample at $2000 \mathrm{rpm}$, creating a uniform coating above the metallic patterns. Finally, the sample is dipped into a solution of EDT in ethanol for $60 \mathrm{~s}$ followed by a rinse in ethanol to strip the NCs from their native OA ligands and leave them instead cross-linked with shorter molecular chains.

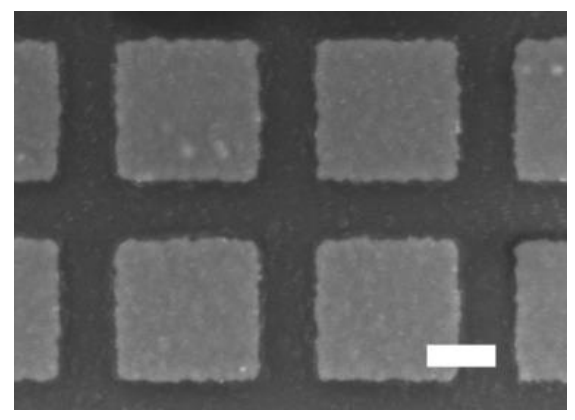

Figure S7. Scanning electron micrograph of the Au patterns. Scale bar $200 \mathrm{~nm}$. 


\section{Photoluminescence experiments}

All the PL experiments are performed with a customized BX51WI microscope from Olympus. Except for the results presented on Figure S11, we pump the sample with a continuous HeNe laser at $633 \mathrm{~nm}$ that is filtered with a BG40 filter from Thorlabs to remove the parasitic spontaneous emission in the near-infrared. This laser is focused to a micron-size spot using a 50X objective. For Figure S11, we have replaced this localized excitation by a wide field illumination scheme that excites a macroscopic part of the $200 \times 200 \mu \mathrm{m}^{2}$ sample. This wide field pumping, covering a circular area with a diameter of $90 \mu \mathrm{m}$, is realized with the same objective and the wide field illuminator of our microscope. The light source attached to this illuminator is a tungsten lamp that is filtered to exclude the wavelengths outside the range $400 \mathrm{~nm}-800 \mathrm{~nm}$.

Our 50X objective (reference LCPLN50XIR) has a numerical aperture of 0.65 and it is also used to collect the infrared PL signal. A dichroic mirror (Thorlabs DMLP950R) is used to separate the IR signal above $950 \mathrm{~nm}$ from the visible pump. The remaining visible light that has not been filtered by the dichroic mirror is subsequently blocked with an RG780 longpass filter. The PL is analyzed with an Acton SP2356 imaging spectrograph coupled to a NIRvana InGaAs camera from Princeton Instruments (Figure S8).

- To record a PL spectrum, a $200 \mu \mathrm{m}$ slit is placed at the spectrograph entrance. Light entering the spectrograph is then dispersed with a 85 groove $/ \mathrm{mm}$ plane rule reflection grating. The raw spectra are then corrected by taking into account the transfer functions of the $50 \times$ objective and the grating.

- To record a dispersion relation, the same configuration is used but an additional Bertrand lens is added in the optical path in a $4 \mathrm{f}$ configuration so as to image the back focal plane of the objective. The function of this lens is to Fourier-transform the signal, which is therefore analyzed in the reciprocal space. 
- To record a back focal plane image, the reflection grating within the Acton SP2356 spectrograph is replaced by a flat silver mirror.

- For the polarization-resolved experiments, a wire grid polarizer (WP25M-UB from Thorlabs) is inserted in the optical path. We have carefully checked the effect of every optical elements on the polarization state of the PL signal, to make sure that no artifact affects the measurements.

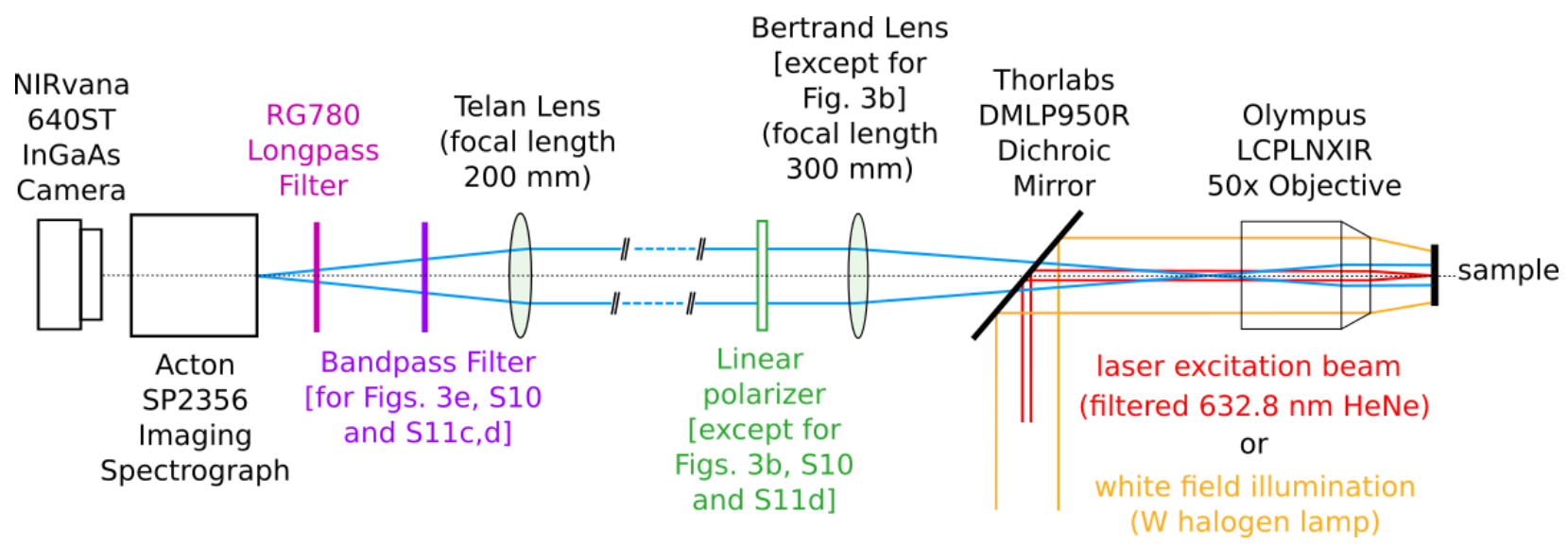

Figure S8. Schematic of the PL setup.

\section{Photocurrent measurements}

The interdigitated samples are biased at $7 \mathrm{~V}$ and illuminated with a $15 \mathrm{X}$ reverse Cassegrain objective using a water-cooled $150 \mathrm{~W}$ incandescent tungsten light source passing through a Fourier Transform Infrared (FTIR) spectrometer (Invenio-R from Bruker). The photocurrent that flows between the electrodes is amplified using a Femto DLCPA-200 amplifier, converted into a voltage and plugged back into the FTIR electronics. By normalizing the data with the spectrum measured with a calibrated 818-ST2 Ge photodiode from Newport, one obtains the responsivity of the sample. 


\section{Supplementary experiments}

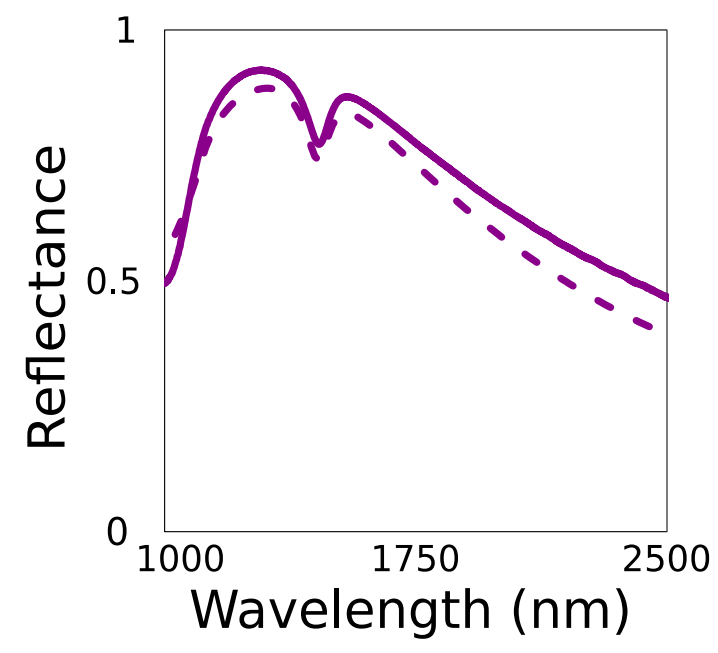

Figure S9. Experimental and simulated reflectance spectra of the sample studied in the main text, which consists in a periodic array of square Au nanoparticles coated with 2-3 layers of PbS NCs (continuous and dashed purple curves, respectively). This plot shows the same spectra as in Figure 3a, but over an extended range of wavelengths.

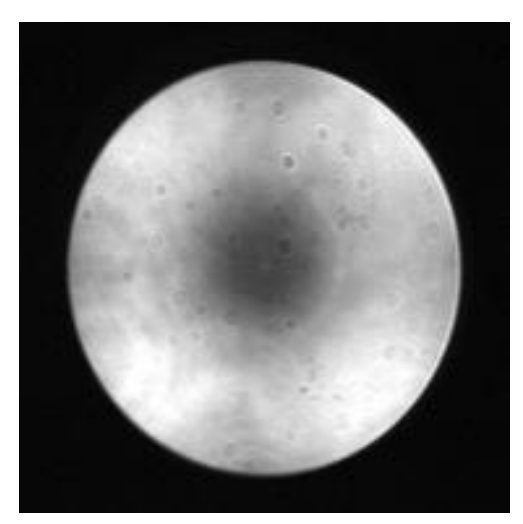

Figure S10. Back focal plane at $1450 \mathrm{~nm}$ without the linear polarizer inserted in the beam path. As in the main text, the experiment is realized by pumping the structure with a diffraction-limited $633 \mathrm{~nm}$ laser spot. 


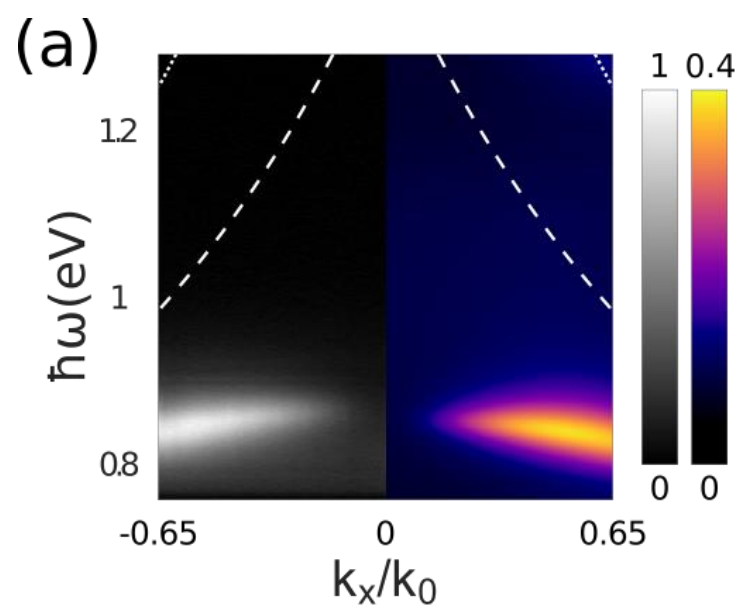

(c)
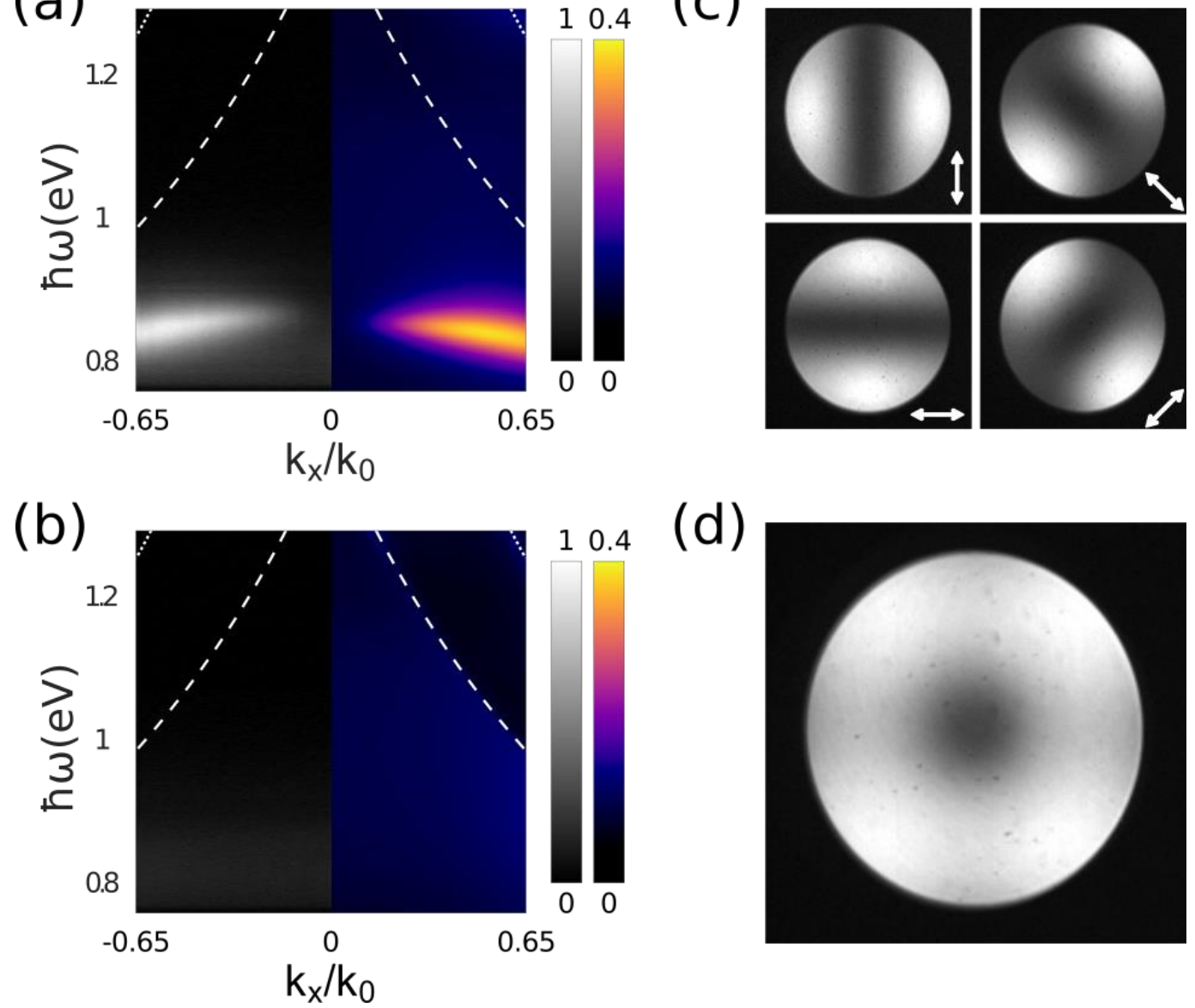

(d)

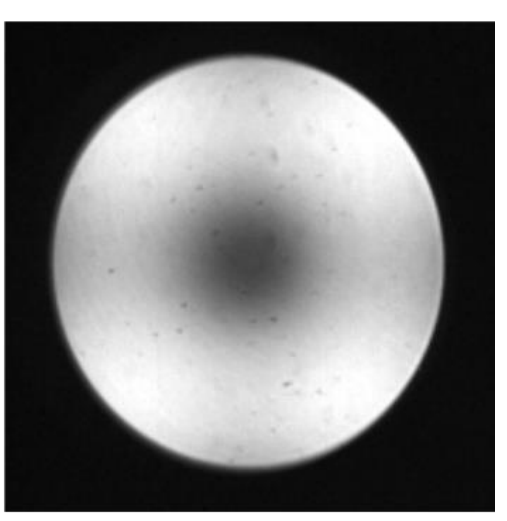

Figure S11. PL experiments realized with a wide field pumping. The sample is the same as the one studied on Figures 3 and S9-S10, except that the pumping is not realized with a diffraction-limited laser spot but by a white-light illumination scheme covering a macroscopic part of the sample (see page 10 of the present document for experimental details). Panels (a)-(b)-(c) plot the same quantities as those plotted on Figure 3c3d-3e. Panel (d) plots the same quantity as in Figure S10. The results are essentially the same as those obtained with the diffraction-limited laser pumping. In fact, the wide field illumination creates an even nicer set of data because it averages the response of the array over a wide area. 

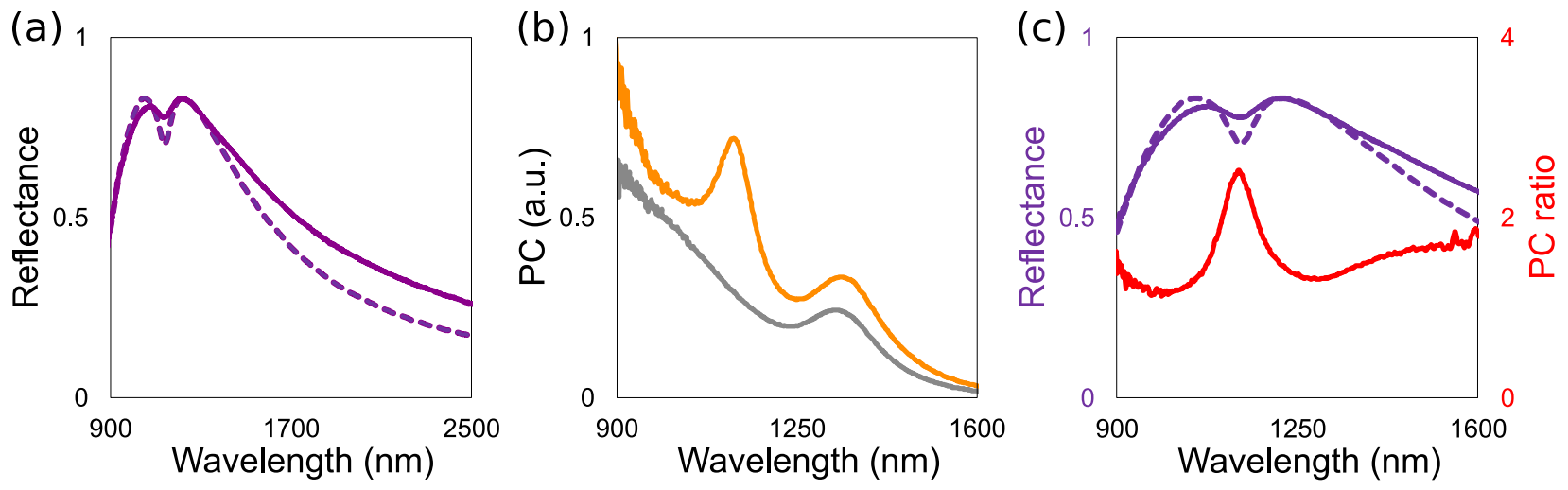

Figure S12. (a) Experimental and simulated reflectance spectra of a metasurface with a period $\mathrm{P}=500 \mathrm{~nm}$ and square $\mathrm{Au}$ nanoparticles with a side of $300 \mathrm{~nm}$, coated with a thin layer of PbS NCs (continuous and dashed purple curves, respectively). (b) Photocurrent spectra of PbS NC photoconductive detectors with (yellow curve) and without (gray curve) such a metasurface inserted between the interdigitated electrodes. The ratio between these two spectra is the red curve of panel (c), which also features a zoom of the curves already plotted on panel (a).

\section{Supplementary references}

(1) Lynch, D. W.; Hunter, W. R. Comments on the Optical Constants of Metals and an Introduction to the Data for Several Metals. In Handbook of Optical Constants of Solids; Elsevier, 1997; pp 275-367. https://doi.org/10.1016/B978-012544415-6.50015-7.

(2) Shalaev, V. M.; Cai, W.; Chettiar, U. K.; Yuan, H.-K.; Sarychev, A. K.; Drachev, V. P.; Kildishev, A. V. Negative Index of Refraction in Optical Metamaterials. Opt. Lett. 2005, 30 (24), 3356. https://doi.org/10.1364/OL.30.003356.

(3) Zhang, S.; Fan, W.; Panoiu, N. C.; Malloy, K. J.; Osgood, R. M.; Brueck, S. R. J. Experimental Demonstration of Near-Infrared Negative-Index Metamaterials. Phys. Rev. Lett. 2005, 95 (13), 137404. https://doi.org/10.1103/PhysRevLett.95.137404.

(4) Kelly, K. L.; Coronado, E.; Zhao, L. L.; Schatz, G. C. The Optical Properties of Metal Nanoparticles: The Influence of Size, Shape, and Dielectric Environment. J. Phys. Chem. B 2003, 107 (3), 668-677. https://doi.org/10.1021/jp026731y. 